\title{
Delivering power
}

\section{Bill Shiner}

\section{With its high wall-plug efficiency and record-breaking power output, the fibre laser has made the use of lasers in manufacturing more acceptable and cost-effective.}

Fibre lasers have developed rapidly over the past several years. Single-mode lasers with powers of around $10 \mathrm{~W}$ were first introduced in 1996, and today multimode fibre lasers with powers that exceed $50 \mathrm{~kW}$ are available. Owing to significant improvements in performance since their introduction, fibre lasers are now an accepted laser technology in materials processing applications and are rapidly expanding the roles they have. The majority of fibre lasers are ytterbium-doped lasers operating at wavelengths of $\sim 1 \mu \mathrm{m}$, serving as replacements for or alternatives to both YAG-crystal and $\mathrm{CO}_{2}$-gas lasers.

Like conventional solid-state lasers based on doped laser crystals, fibre lasers rely on glass doped with a rare earth element (ytterbium or erbium being the most common) as a gain medium. The key difference is that the geometry of the gain medium is now a thin length of fibre rather than a small rod. This brings many benefits in terms of deployment and convenience of operation, as well as for thermal management and power scaling. Fibre lasers are optically pumped, usually by high-power semiconductor laser diodes.

The technology behind fibre lasers has made their use in manufacturing more acceptable and cost-effective than previous laser technologies, and their high wall-plug efficiencies (around 30\%) greatly reduce the electrical operating costs associated with an industrial laser. Fibre lasers are also maintenance-free. This allows the technology to compete, on a cost-of-manufacturing basis, with many of the more conventional materials processing technologies. Fibre lasers are also extremely rugged and compact, allowing their deployment in mobile and outdoor applications for which lasers could never have previously been considered. Fibre lasers are also now available at very high power levels, with systems offering output powers up to $50 \mathrm{~kW}$ and thus greatly expanding the applications of the technology. The excellent beam quality of fibre lasers has expanded the use of long-focal-length lenses that are required for many remote welding applications; for example, fibre lasers can produce a $500 \mu \mathrm{m}$ focused spot at a distance

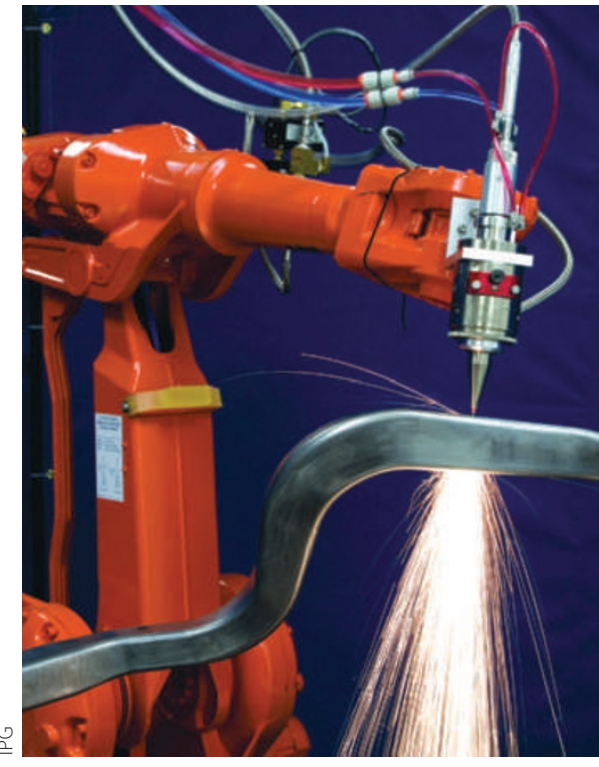

The fibre laser has become a valuable materials processing tool, with output powers reaching $10 \mathrm{~kW}$ for single-mode devices and $50 \mathrm{~kW}$ for multimode devices.

of $4 \mathrm{~m}$. These long focal lengths are required for high-speed welding applications that use robots or high-speed scanning systems.

In 2009, industry saw the increased acceptance of fibre lasers, particularly in the number of sales to companies that manufacture standard two- and threeaxis sheet-metal cutting machines. This achievement, in a market previously totally dominated by the $\mathrm{CO}_{2}$ laser, marks the beginning of a shift that is accelerating towards the mass-adoption of fibre lasers. The advantages are clear: fibre lasers can cut thin materials faster than, and with a quality equal to or superior than, $\mathrm{CO}_{2}$ lasers. Furthermore, the fibre-based method of delivery eliminates costly beam paths and alignment requirements, and produces the same spot size over the entire cutting envelope.

The fibre laser has also made significant progress in the extremely competitive automotive market, where it is exploited for single-use welding and cutting applications. These applications include the cutting of hydro-formed rails and high-strength body sheet metal, as well as the welding of tailored blanks and seat structures, many of which are manufactured from high-strength steel. The acceptance of fibre lasers has been aided by the ease at which they can be integrated into robots, and the elimination of expensive helium cover gas for welding. High-power fibre lasers with outputs of $10-20 \mathrm{~kW}$ have been deployed for deep-penetration welding in the nuclear, railroad, shipbuilding and land-based turbine industries. Many of these applications previously required an electron-beam welding machine operating in vacuum, but instead can now weld in air using an ultrahigh-power fibre laser. Another growth industry for fibre lasers is the welding of the new battery designs for the electric car industry. This application includes both the hermetic sealing and welding of the copper contacts that combine individual cells. Low-power fibre lasers in the range of $5 \mathrm{~W}$ to $1 \mathrm{~kW}$ - both pulsed and continuous wave - have gained significant market share over previous technologies in the computer, solar, medical device and marking industries, with more than 6,000 units being shipped worldwide every year.

Two recent products introduced to the market are a $10 \mathrm{~kW}$ single-mode fibre laser and pulsed and continuous-wave green $(532 \mathrm{~nm})$ fibre lasers. Such fibre lasers are continuing to increase their double-digit market share in the materials processing sector, with laser output powers now available from $5 \mathrm{~W}$ to $50 \mathrm{~kW}$. These lasers also are being used for many other applications such disarming land mines at great distances, shooting down airborne devices, light detection and ranging mapping applications, optical pumping, skin wrinkle reduction and surgical applications. In the future, the brightness and compactness of fibre lasers will continue to improve, opening up many new applications and, most importantly, reducing the cost of ownership.

Bill Shiner is vice president of industrial at IPG Photonics, 50 Old Webster Road, Oxford, Massachusetts 01527, USA.

e-mail:bshiner@ipgphotonics.com 